\title{
Desarrollo social en el Trastorno del Espectro Autista (TEA) en niños entre 3 y 6 años de edad*
}

\author{
Social development in autism spectrum disorder In children between 3 \\ and 6 years of age
}

Maribel Lanchero Bello ${ }^{1}$, Marta Pérez Correa ${ }^{1}$, Paola Flórez Vergara ${ }^{1}$

${ }^{1}$ Estudiante Especialización desarrollo Integral en Infancia y adolescencia. Corporación Universitaria Iberoamericana sofiadelcarmen31@gmail.com

* Artículo original derivado del proyecto de trabajo de grado titulado: Desarrollo social en el trastorno del espectro autista en niños entre 3 y 6 años estrato socioeconómico bajo en la ciudad de Cartagena de Indias Departamento de Bolívar, desarrollado en la Corporación Universitaria Iberoamericana; Especialización en Desarrollo Integral de la Infancia y la Adolescencia, (Bogotá-Colombia).

Recibido: 15/feb/2021 Revisado: 30/abr/2021

Aceptado: 30/may/2021 Publicado: 30/jul/2021

Resumen El trastorno del espectro autista (TEA) es una discapacidad del desarrollo que puede inducir a problemas sociales de comunicación y conducta de forma significativa, las destrezas de aprendizaje, pensamientos y resolución de problemas. Es importantes empezar a intervenirlas una vez se confirme el diagnóstico del trastorno, algunas necesitan mucha ayuda en la vida diaria, mientras que otras necesitan menos. El presente estudio es de tipo Cualitativo fenomenológico, tiene como objetivo comprender la experiencia, entorno al desarrollo social en el trastorno del espectro autista en niños entre 3 y 6 años de edad de estrato socioeconómico bajo, en la ciudad de Cartagena de Indias - Bolívar, para lo cual se emplearon las entrevistas a profundidad, se obtuvo como resultado una relación impactante y de alto significado entre el desarrollo social y las experiencias de la vida diaria que estos niños se enfrentan, ocasionando con frecuencia frustraciones por no ser entendido, comprendido y satisfacer sus deseos y necesidades, al igual que una correlación significativa entre los niveles socioeconómicos y las estrategias de direccionamiento, acompañamiento, oportunidad y acceso a intervenciones intensivas pertinentes.

Palabras clave desarrollo social; autismo; comportamiento; lenguaje; habilidades.

Abstract Autism spectrum disorder (ASD) is a developmental disability that can induce social, communication, and behavioral problems in a significant way, learning skills, thoughts and problem solving are important to start intervening once the diagnosis of the disorder is confirmed, because some need a lot of help in daily life, while others need less. The present study is of phenomenological Qualitative type, aims to understand the experience around social development in autistic spectrum disorder in children between 3 and 6 years of age of low socioeconomic strata in the city of Cartagena de India, Bolivar, for which in-depth interviews were used, which resulted in an impactful and high-meaning relationship between social development and the daily life experiences these children face, often causing frustrations at not being understood, understood and satisfy their desires and needs, as well as a significant correlation between socio-economic levels and the strategies of addressing, accompanying, opportunity and access to relevant intensive interventions.

Keywords social development; autism; behavior; language; skills.

Teknos Revista Científica. | Volumen 21 No.1 - diciembre 2021 | ISSN 1900-7388 (papel) | ISSN 2539-2190 (digital)

DOI: https://doi.org/10.25044/issn.2539-2190 


\section{Introducción}

En los últimos cinco años en la ciudad de Cartagena - Bolívar, se ha reportado un crecimiento importante de trastorno del espectro autista (TEA) evidenciando una afectación significativa en esta población, debido a que no se realiza en los niños, un adecuado proceso de intervención terapéutica donde se involucren respuestas de estímulo hacia los demás, experiencias compartidas con los otros, dificultando la comunicación afectiva y manifestaciones emocionales, al no mostrar empatía con su entorno y no saber compartir sus emociones con los demás niños.

Roldan, L. (2016) define el Autismo como un conjunto de discapacidades del desarrollo, que pueden causar impactos sociales, de comunicación y problemas del comportamiento. Los niños con TEA procesan la información del cerebro de forma diferente al resto de personas. El autismo afecta a los niños de diferentes formas y puede variar de leve a grave.

El autor también afirma que los niños con autismo comparten algunos síntomas, como las dificultades con la interacción social, pero existen diferencias entre los mismos, porque el grado de afectación varía; además de que pueden existir otros tipos de problemas. Un niño con autismo se puede empezar a intuir después de los seis meses de edad, pero es a partir de los 3 años, cuando pueden mostrar los indicios de problemas y se puede empezar a pensar en un diagnóstico claro. El autismo no entiende de razas, ni etnias ni de grupos socio-económicos, y aunque es más común entre niños que niñas, puede afectar a ambos sexos por igual. Así mismo la mayoría de infantes con autismo muestran diferencias en la comprensión emocional, porque cada niño es un mundo y no se puede generalizar a todos por igual. Los niños con autismo no suelen mostrar empatía con el rostro de las personas y tienen dificultad en las relaciones sociales, juegan de manera aislada con actividades de auto estimulación en lugar de compartir su tiempo con otros.

De esta manera, partiremos de la observación de actividades donde el niño pueda compartir, en un espacio de encuentro con un marco de intercambio, el contexto de la vida cotidiana, de esta manera analizaremos la interacción, el proceso de construcción, organización de las actividades conjuntas y particulares, proceso de cesión y traspaso de control, que operan entren los niños en el transcurso de esta relación, generando momentos auténticos.

No obstante, el proceso de atención, comunicación e interacción social de este grupo poblacional, representa un reto para los padres, cuidadores y profesionales, por lo tanto se requiere del compromiso de la familia como núcleo social principal, y de los diferentes actores públicos y privados en aras de consolidar las estrategias metodológicas que contribuyan a fortalecer las actividades, intervenciones y programas en los diferentes espacios.

Nuestro interés con esta investigación es realizar un análisis a través de la experiencia, que demuestre las múltiples estrategias que se pueden implementar en este grupo poblacional para mejorar la calidad y cualidad de la Interacción social, profundizando los procesos interpsicológicos.

Actualmente, es necesario comprender el desarrollo social de un niño diagnosticado con trastorno del espectro autista (TEA). Por lo tanto, es indispensable hacer un estudio desde el accionar psicológico para indagar que aspectos inician, mantienen, y prevalecen sobre un niño diagnosticado con TEA.

De acuerdo con Cox, D. (1996), Social Development: The Developmental Perspective in Social Welfare, Londres, Sage. El Desarrollo social en los niños entre los 3 y 4 años de edad, "es el proceso por el que aprenden a interactuar con los que están a su alrededor, desde las personas de referencia, con los que establecen el apego cuando eran bebés, pasando por el complejo entramado que compone la sociedad en la que viven".

El Desarrollo social es un proceso que con el transcurso del tiempo conduce al mejoramiento de las condiciones de vida de estos niños, en esta etapa los niños interactúan y juegan juntos, son más independientes, se interesan por experimentar, probar 
Lanchero Bello, M., Pérez Correa, M., \& Flórez Vergara, P. (2021). Desarrollo social en el Trastorno del Espectro Autista (TEA) en niños entre 3 y 6 años de edad. Teknos Revista Científica, 21(1), 46-54.

situaciones y sensaciones nuevas, que logran a través del juego, desarrollar la empatía que se convierte para ellos en una herramienta social y emocional.

De acuerdo con González, C. (2020, pág. 150), American Academia of Pediatrics. El Trastorno del Espectro Autista "es una alteración en el neurodesarrollo en la que se compromete el desarrollo comunicativo, emocional y simbólico del niño, así como la capacidad de establecer relaciones con el adulto y los objetos; en las edades entre 3 y 6 años, se muestran incapaces de mostrar la mirada, postura y la expresión para comunicarse".

Según Gutiérrez, K. (2016). A nivel internacional se ha estimado que la prevalencia de TEA se ha incrementado en los últimos años, pasando de 2/10000 a cifras que oscilan entre 2 y 6.7/1000, lo que significa que hay un caso de TEA por cada 150 - 500 Niños. Así mismo el autor afirma que estudios más recientes reportan un caso por cada 180 niños y de esta manera el TEA se constituye en uno de los trastornos graves más frecuentes que afectan el desarrollo de los niños desde edades tempranas.

En este orden de ideas Roldan, L. (2016), plantea que los niños con autismo, pueden tener diferencias entre sí, pero siguen siendo niños y como tales tendrán sus propios rasgos característicos, por lo cual se debe respetar su mundo interior, no forzarles a hacer cosas que no quieren, establecer rutinas diarias y sobre todo, aceptarles tal y cómo son.

Según datos publicados por la organización mundial de la salud en junio de 2021, se calcula que uno de cada 160 niños tiene autismo, y en otros estudios bien controlados se han registrado cifras notablemente mayores, sin embrago, la prevalencia de autismo en muchos países de ingresos bajos y medios, es en este momento desconocida. En este sentido, y según estos datos, una vez que se haya identificado un caso de autismo, es importante que se le ofrezca al niño y a su familia, información y servicios pertinentes, derivación a especialistas y ayudas prácticas, de acuerdo con las necesidades particulares de cada individuo. Si bien no hay "cura" para el autismo, las intervenciones psicosociales basadas en la evidencia (como la terapia conductual y los programas de capacitación para los padres y otros cuidadores), pueden reducir las dificultades de comunicación y comportamiento social, y tener un impacto positivo en la calidad de vida y el bienestar de la persona.

A nivel nacional, según el Ministerio de Protección Social, los datos estadísticos vinculados a la prevalencia de autismo, refieren una cifra de 30.000 niños diagnosticados con Trastornos del Espectro Autista /2017).

En la ciudad de Cartagena, departamento de Bolívar, se realizó un estudio no experimental de casos y controles de corte transversal, con una muestra conformada por 40 niños (36 - 48 meses), 39 hombres y una mujer, con diagnóstico temprano de TEA y sus cuidadores principales; y 40 niños con desarrollo típico (36 - 48 meses), 21 hombres y 19 mujeres, y sus cuidadores principales. Los niños con diagnóstico de TEA conformaron el grupo clínico (casos) y los niños con desarrollo típico, el grupo de comparación (control), apareados por edad y estrato socioeconómico. Para conformar el grupo clínico, se realizó un muestreo no probabilístico por conveniencia, identificando en la base de datos de los usuarios de las instituciones de salud Rehabilitar IPS y Pediacenter (Cartagena, Colombia), los niños con edades comprendidas entre 18 y 40 meses, con diagnóstico de TEA de acuerdo con los criterios del CIE-10 (Organización Mundial de la Salud -OMS-, 1992) o DSM-IV-TR (American Psychiatric Association -APA-, 2002). El diagnóstico fue realizado previamente por un equipo multidisciplinario conformado por un neuropediatra, un psicólogo clínico, un neuropsicólogo y un fonoaudiólogo. La presencia de cualquiera de las siguientes afecciones se consideró como criterio de exclusión: historia de hipoacusia severa, trastornos genéticos, trastornos metabólicos, síndrome de Guilles de la Tourette, encefalopatía, meningitis y discapacidad intelectual severa, que cursara con síntomas similares a los encontrados en el TEA. Los niños del grupo de control tenían historia de desarrollo neurológico típico y no presentaban antecedentes neurológicos y/o psiquiátricos. Para la selección de este grupo se realizó un muestreo no probabilístico por conveniencia de niños usuarios de un centro de estimulación infantil, 
Lanchero Bello, M., Pérez Correa, M., \& Flórez Vergara, P. (2021). Desarrollo social en el Trastorno del Espectro Autista (TEA) en niños entre 3 y 6 años de edad. Teknos Revista Científica, 21(1), 46-54.

también de la ciudad de Cartagena, Colombia, para comprender la experiencia entorno al desarrollo social, en el trastorno del espectro autista en niños entre 3 y 6 años de edad de estrato socioeconómico bajo, en la ciudad de Cartagena de Indias - Bolívar.

\section{Material y métodos}

\subsection{Tipo de estudio cualitativo - fenomenológico}

La metodología cualitativa de tipo fenomenológica, se orienta hacia la comprensión de las situaciones únicas y particulares, se centra en la búsqueda de significado y de sentido que les conceden a los hechos los propios agentes, y en cómo viven y experimentan ciertos fenómenos o experiencias, el grupo de niños a los que investigamos.

Población: Niños con Trastorno de Espectro Autista confirmado entre 3 y 6 años de edad de estrato socio económico bajo en la ciudad de Cartagena de Indias - Bolívar, que asisten a una fundación para recibir atención integral.

Muestra: Es un muestreo no probabilístico por conveniencia, donde se seleccionaron 10 niños entre 3 a 6 años de edad, que asisten a la fundación.

\subsection{Técnicas para la recolección de la información}

La principal herramienta de recolección de información fue la entrevista a profundidad, debido a que: "La entrevista cualitativa posibilita la recopilación de información detallada en vista de que la persona que informa comparte oralmente con el investigador aquello concerniente a un tema específico o evento acaecido en su vida" como plantea (en Vargas, J. 2012, p. 123).

La entrevista en profundidad según Rincón et al. (1995), "está compuesta por preguntas abiertas, debido a que estas no están prefijadas, entonces el entrevistado debe construir una respuesta elaborada y le permite al entrevistador guiarse y adaptarse a la situación actual de la entrevista, es necesario que el investigador tenga más conocimiento y experiencia en este tipo de entrevista, ya que la información resulta ser más compleja de analizar y de sistematizar" (Citado por Vargas, 2012, pg. 126).

\subsection{Procedimientos y análisis de datos}

Se realizó entrevista a 10 niños, 8 niños, 2 niñas, en la cual se indaga acerca de los lugares donde prefieren ir de vacaciones, lo que les produce felicidad, lo que quieren ser cuando crezcan, su reacción al perderse en un centro comercial, su gusto por jugar con amigos o tener amigos, su juego favorito, su predilección de jugar con los papas, la tarea de más interés en el colegio, su actitud hacia la tristeza, su diversión al jugar con diferentes juguetes y lo que más le incomoda. La entrevista se realiza y se graba bajo el permiso de los padres de cada niño participante y luego se transcribe la relación de opiniones elegidas de acuerdo con nuestro esquema de conceptualización de opiniones, junto con las anotaciones pertinentes relativas a los incidentes del proceso y demás manifestaciones. Fue evidente anotar tanto las palabras literales como los apartados acumulados de incidentes ocurridos y de la comunicación no verbal. Luego fueron analizados sistemáticamente, se obtienen los códigos, se agrupan por familias, para un análisis e interpretación detallada y profunda. Acerca de la experiencia entorno al desarrollo social, fueron identificados en las transcripciones de las entrevistas 10 códigos significativos, al realizar de frecuencia de las palabras más usadas por los niños al responder las diferentes preguntas, teniendo en cuenta que una de las características en el desarrollo social en los niños con este diagnóstico, son contestaciones cortas a los interrogantes. Los códigos se han agrupados en cuatro familias, para un mejor análisis y comprensión. Su distribución se presenta en la sección de resultados. 
Lanchero Bello, M., Pérez Correa, M., \& Flórez Vergara, P. (2021). Desarrollo social en el Trastorno del Espectro Autista (TEA) en niños entre 3 y 6 años de edad. Teknos Revista Científica, 21(1), 46-54.

\subsection{Consideraciones éticas}

De acuerdo al Acuerdo 11 de la Resolución 008430 de 1993 del Ministerio de Salud en el Artículo 8, esta investigación protegerá los datos e informaciones personales de los participantes. Teniendo en cuenta el artículo 9, esta investigación no representa riesgos para la vida o seguridad de los participantes. Conforme al acuerdo 10, los líderes y colaboradores del proyecto se comprometen a identificar los tipos de riesgo a los que pueden estar expuestos los participantes de esta investigación. De acuerdo con el Acuerdo 11, literal (a) esta investigación trabajará con personas, pero no se le realizará ningún tipo de intervención o modificación intencionada de las variables biológicas, fisiológicas, psicológicas o sociales de los individuos que participan en el estudio, entre los que se consideran: revisión de historias clínicas, entrevistas, cuestionarios y otros en los que no se le identifique ni se traten aspectos sensitivos de su conducta. Por último, se velará por el bienestar de los participantes cumpliendo con los artículos 12, 13, 14 y 15 de la misma resolución.

\section{Resultados}

Continuando con el desarrollo de la investigación, se abordan las bases teóricas, con el fin de describir conceptos, ideas y sentidos que nos permitan dar respuestas ¿Cuál es la experiencia en torno al desarrollo social en el trastorno de espectro autista en niños entre 3 y 6 años del estrato socioeconómico bajo, en la ciudad de Cartagena de Indias - Bolívar?, se han identificado en las transcripciones de las entrevistas 10 códigos significativos, teniendo en cuenta que una de las características en el desarrollo social en los niños con este diagnóstico, son respuesta cortas a los interrogantes. Los códigos se han agrupados en 4 familias, para un mejor análisis y comprensión. El código encontrado con mayor frecuencia es el amor, dado que estos niños al jugar con sus padres y amigos se sienten amados y comparten con amor, este código se encuentra dentro de la familia de confianza y seguridad, dado que cuando los niños están con sus padres se sienten protegidos y con seguridad para desenvolverse. La felicidad también fue encontrada con una frecuencia de 20 repeticiones, la cual se encuentra dentro de la familia de satisfacción personal, dado que los niños expresan felicidad al jugar con sus padres, amigos, sus juguetes favoritos e irse de viaje, dentro de la categoría de frustración se destaca el nerviosismo con una frecuencia de 20 repeticiones, dado que al perderse en un centro comercial expresan que se sienten muy nerviosos. Los demás códigos y sus familias se detallan en la tabla 1.

Tabla 1. Distribución de códigos

\begin{tabular}{ccc}
\hline Códigos & $\begin{array}{c}\text { Total de } \\
\text { códigos }\end{array}$ & Familia de códigos \\
\hline Felicidad & 20 & Satisfacción Personal \\
Viajes de & 10 & \\
Vacaciones & 10 & \\
Amistad & 10 & Confianza y \\
\hline Amor & 22 & Seguridad \\
Protección & 15 & \\
\hline Juegos de & 10 & Frubito social \\
Tecnología & & \\
Juegos de & 10 & \\
Imitación & & \\
\hline Llorar & 10 & \\
Nerviosismo & 20 &
\end{tabular}

Dada la frecuencia de los códigos, se puede evidenciar en este grupo, niños que en la medida en la que ellos se sientan felices, amados y protegidos, sus habilidades sociales se podrían desarrollar con mayor rapidez y efectividad en los diferentes entornos en los que se desenvuelven.

Un factor que afecta el desarrollo social comprobado en los casos de esta investigación, es la frustración demostrada a través del nerviosismo de los niños cuando se encuentran en situaciones de angustia, al no poder encontrar una solución inmediata a su problema.

\section{Codificación Axial}

En el análisis textual realizado a las respuestas de los niños, a través de la frecuencia de las repeticiones, 
Lanchero Bello, M., Pérez Correa, M., \& Flórez Vergara, P. (2021). Desarrollo social en el Trastorno del Espectro Autista (TEA) en niños entre 3 y 6 años de edad. Teknos Revista Científica, 21(1), 46-54.

se pueden ver las relaciones entre los códigos y sus familias, obtenidas en la codificación abierta con la resistencia existencial, esto nos permite construir nuestra propia teoría, acerca de las experiencias en torno al desarrollo social, en el trastorno del espectro autista. La satisfacción personal en este grupo de niños se encuentra basada en la confianza y seguridad generada por el amor y la protección que encuentran en sus padres y contextos sociales, a su vez este estado les proporciona mejor calidad de vida, sintiéndose feliz cada vez que experimentan momentos en disfrute y gozo, como es compartir tiempo suficiente y pleno con sus padres, emprender viajes a lugares de su agrado donde exploren y observen nuevas cosas. Los juegos de tecnología y de mi imitación constituyen para ellos un ámbito de relaciones sociales, debido a que se sumergen $y$ actúan como si estuvieran en el mundo real, causando un impacto social.

Por otro lado, tenemos la frustración que podíamos decir es un estado casi que habitual, debido a situaciones básicas de deseo y necesidad que no saben expresar para ser entendidos y comprendidos, de igual manera hay situaciones particulares en las que no saben cómo actuar y sienten una gran imposibilidad que genera llanto y nerviosismo, intranquilidad, agresividad, en fin, conductas inadecuadas provenientes del estado de frustración en el que se encuentren. En el caso particular de esta investigación se comprobó que al realizar la pregunta sobre ¿Qué haría si se perdiese en un centro comercial?, en tres casos los niños evidenciaron no entender la pregunta, por lo cual se le repite, sin embargo, ellos al no comprender, muestran un comportamiento inapropiado como es gritar, llorar, expresar "no quiero", uno de los niños muestra una conducta adicional que es taparse los oídos y 7 de los 10 niños, no establecieron contacto visual.

En cuanto a la experiencia relacionada con el ámbito social, se demostró que los niños mostraban interés y atracción por los juegos de imitación y tecnología, tales como correr patear, jugar con carros utilizando los sonidos de algunos medios de transporte, juegos de robot, utilizando aplicaciones como "cailou", "kidEcats", "cuidado de animales, "Doctor", "amongus", entre otros.

Cabe destacar que 8 de los 10 niños entrevistados, han tenido intervención terapéutica en diferentes IPS de la ciudad de Cartagena, cinco de los cuales bajo la metodología ABA y tres con terapia convencionales.

Todas estas experiencias mencionadas se muestran en la figura 1

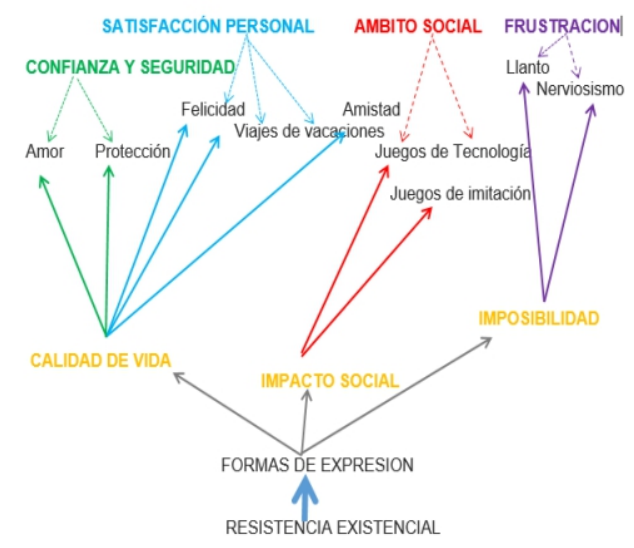

Figura 1. Esquema Teórico de Relación Teoría-Código

Estos resultados se relacionan con lo expresado en Roldan, L. (2016), dado que los niños presentaron problemas de comportamiento como gritar y taparse los oídos, lo cual ocurre porque procesan la información de manera diferente teniendo en cuenta que la primera instrucción dada no fue comprendida.

Asimismo, se comprobó lo expresados por González, C. (2020), con respecto a la falta de contacto visual en la mayoría de los entrevistados al responder las preguntas.

\section{1 contextos de la vida de un niño con TEA}

De acuerdo con los niños entrevistados, se puede decir que muestran la confianza de sentirse listos para hacer frente a las experiencias de la vida, siendo este un aspecto muy importante, porque contribuye al acercarse con los demás niños, personas y entorno en general, al igual que al acceso de oportunidades, a ser creativos y enfrentar experiencias nuevas.

En este sentido se pueden encontrar 3 grupos, los niños de 3 a 4 años, quienes manifestaron a través del 
Lanchero Bello, M., Pérez Correa, M., \& Flórez Vergara, P. (2021). Desarrollo social en el Trastorno del Espectro Autista (TEA) en niños entre 3 y 6 años de edad. Teknos Revista Científica, 21(1), 46-54.

lenguaje corporal (no verbal), sentirse protegidos cuando juegan con sus padres y comparten con amigos que le brindan apoyo y pueden entender lo que ellos quieren expresar.

En el segundo grupo se encuentran los niños de 4 a 5 años, teniendo en cuenta que su lenguaje no es fluido, manifestaban corporal y verbalmente su seguridad, cuando juegan con padres y pares conocidos.

Finalmente, en el tercer grupo, de 5 a 6 años, se observa una mayor madurez en el reconocimiento de la seguridad y confianza al jugar con padres y pares conocidos, de forma verbal y no verbal.

Para una mejor comprensión se ilustran las dinámicas relacionales en estos contextos figura 2 .

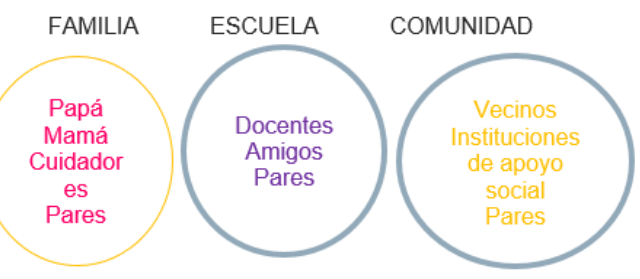

Figura 2. Contextos de la vida de un Niño con TEA

\subsection{Mantenedores de la Resistencia Existencial}

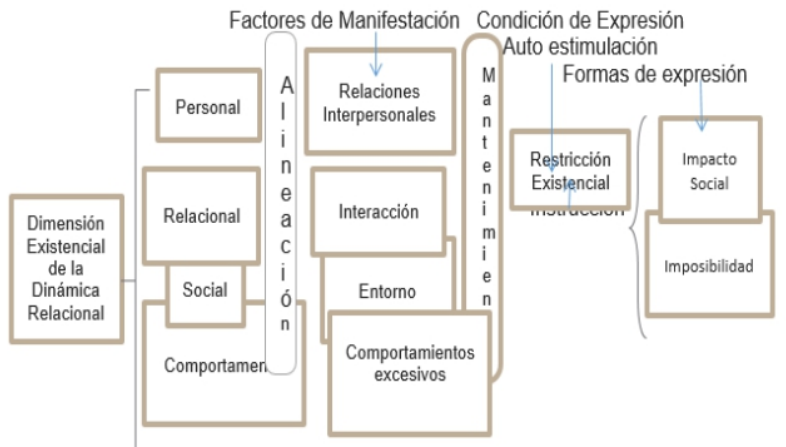

Figura 3. Esquema de los mantenedores, Formas de Expresión y manifestaciones de la Resistencia Existencial. Fuente: Elaboración propia de los autores (2021)

La Resistencia Existencial se inicia a partir de dos condiciones de expresión, formadas a través del auto estimulación y las instrucciones impartidas. Estas dos condiciones van siendo implantadas en las dinámicas relacionales de los niños desde su infancia, siendo cerrada la posibilidad de formarse existencialmente; en el diagnóstico temprano va asumiendo su resistencia existencial como un desinterés en el juego colectivo, actividades recreativas, sociales y se van alejando del entorno que los rodea.

El Autismo es una ruptura del proceso normal del desarrollo, que ocurre en los dos primeros años de vida, se manifiestan problemas de lenguaje, juegos, conocimientos, funciones sociales y adaptación. Es aquí donde esta población de niños, nos permite comprender no solo como se ven expresadas las formas de la resistencia existencial, sino que nos lleva profundamente a los factores que mantienen ésta misma, a través de sus relatos que han sido de satisfacción personal, confianza y seguridad, ámbito social, frustración, las cuales se han orientado a mostrar las condiciones que dan la resistencia existencial, expresadas en la calidad de vida, impacto social e imposibilidad.

\section{Discusión}

El aspecto de los niños con TEA, se diferencia de otros niños, pero es posible que se comuniquen, interactúen, se comporten y aprendan de forma distinta a los demás. Las capacidades y habilidades de aprendizaje, pensamiento y resolución de problemas de los niños con TEA pueden variar; hay desde muy altos niveles de capacidad (dotadas, en español o inglés) y otros que tienen muchas dificultades. Algunos necesitan mucha ayuda en la vida diaria, mientras que otros necesitan menos. Esto se comprobó en los niños entrevistados, dado que cada uno demostró una capacidad intelectual diferente, evidenciándose en la profundidad de las respuestas, así como comportamientos diversos como frustración, al no entender la pregunta, llanto y taparse los oídos, también se registraron casos donde los niños tuvieron la capacidad de mantenerse tranquilos sin presentar comportamientos disruptivos, aun cuando no entendían la pregunta. 
Lanchero Bello, M., Pérez Correa, M., \& Flórez Vergara, P. (2021). Desarrollo social en el Trastorno del Espectro Autista (TEA) en niños entre 3 y 6 años de edad. Teknos Revista Científica, 21(1), 46-54.

En el objetivo general que se planteó en esta investigación, se pretendió entender la experiencia, entorno al desarrollo social en el trastorno del espectro autista en niños entre 3 y 6 años, de estrato socioeconómico bajo en la ciudad de Cartagena de Indias - Bolívar. La discusión se centró en aquellos aspectos más relevantes que se han extraído de los resultados obtenidos, de elementos específicos que muestran logros y nuestras aportaciones.

En lo planteado en el marco teórico, los niños con TEA tienen poca comunicación verbal y no verbal para buscar la interacción con otros, puede que si se comunican con los demás, no usen la gesticulación y que tenga una expresión emocional ausente, además prestarán menos atención a las manifestaciones emocionales de los demás por lo que al no darles importancia, la comunicación afectiva se verá gravemente perjudicada. Esto aún será más problemático por un lado, al no mostrar empatía por los demás o a no saber compartir las emociones, y por el otro la sensación de rechazo de las personas, causando gran impacto a nivel familiar, en algunos casos crisis intrafamiliares que conllevan a depresión, para lo cual se hace necesaria, la ayuda profesional con apoyo y asesoramiento para hacer frente al reto que implica la convivencia con un niño afectado con TEA. En el caso de esta investigación a pesar de no estar dirigida a los padres, si se pudo tener interacción con ellos donde expresaron las crisis que han tenido que enfrentar a nivel familiar y personal, al conocer el diagnóstico, donde referían que enfrentaron etapas de no aceptación, depresión en alguno de los miembros de la familia y tuvieron que ser intervenidos de manera profesional.

La solución al problema planteado en los niños con TEA, es centrarlo a la terapia de estimulación integral, que hace parte del comportamiento excesivo, estas auto estimulaciones son: táctiles, visuales, verbales, vestibular auditivas, con objetos, las cuales buscan que los niños logren compartir sus experiencias con los demás, se sientan satisfechos en las necesidades $y$ deseos y finalmente obtener una excelente calidad de vida para el paciente y para su familia. En este momento existen formas de atención integral con diferentes terapias que favorecen mucho el proceso de inclusión para ellos.

\section{Conclusiones}

Se puede concluir que los niños con TEA entrevistados se sienten protegidos cuando tienen el acompañamiento de los padres y la aceptación de sus pares, sin embargo, también se pudo identificar que hay dificultad en el acercamiento con personas regulares, en este caso el entrevistador.

Así mismo, se comprobó que la interacción social del niño se puede ver afectada cuando él no comprende totalmente la pregunta realizada o la instrucción dada, manifestándose en comportamientos disruptivos, como frustración, llantos y taparse los oídos. Estas conductas según los elementos teóricos analizados, podrían dificultar los procesos de enseñanza- aprendizaje.

En este mismo sentido, se pudo identificar que socialmente los niños con TEA pueden desarrollar habilidades de juego, siendo más atractivos los de carácter tecnológico o de imitación, esto puede propiciar la adquisición de nuevas habilidades si es monitoreado correctamente, sin embargo, si no recibe supervisión puede acarrear adicción y problemas de socialización.

Finalmente se pudo deducir, que cuando el cuidador está presente en las etapas del desarrollo del niño, existe mayor probabilidad de desarrollar afectividad e interacción social positiva con el entorno, tal como se evidenció en los niños entrevistados que manifestaron sentir los lazos de amor de sus familiares y pares.

\section{Referencias}

Cox, D. (1996). Desarrollo social: la perspectiva del desarrollo en el bienestar social por James Midgley 1995, Sage Publications, Londres. Niños Australia, 21 (1), 31-31. doi: 10.1017 / S1035077200004776.

González, M. (2020). Desarrollo de la imaginación narrativa creadora en un niño con Trastorno del Espectro Autista. Summa Psicológica UST. Vol. 17, N², 149 165. 
Lanchero Bello, M., Pérez Correa, M., \& Flórez Vergara, P. (2021). Desarrollo social en el Trastorno del Espectro Autista (TEA) en niños entre 3 y 6 años de edad. Teknos Revista Científica, 21(1), 46-54.

Gutiérrez, K (2016). Identificación temprana de trastornos del espectro autista. Acta Neurológica Colombia volumen $32 \mathrm{~N}^{\circ} 3,238-247$.

Organización mundial de la salud (1 junio de 2021). Trastornos del espectro autista. https://www.who.int/es/news-room/factheets/detail/autism-spectrum-disorders
Roldan, L (2016). El autismo y el desarrollo social. Etapa infantil.

https://www.etapainfantil.com/autismo-desarrollosocial

Vargas J. (2012). La Entrevista en la investigación cualitativa. Nuevas tendencias y retos. Revista electrónica calidad de la educación superior. ISSN 16594703 Vol. 3 N¹. 120-139 